\title{
Depth-reversal-free three-dimensional display by adjusting the light field alignment
}

\author{
Songlin Xie, Xinzhu Sang*, Peng Wang, Nan Guo, Zhidong Chen, Xunbo Yu, \\ Binbin Yan, Kuiru Wang, and Chongxiu Yu \\ State Key Laboratory of Information Photonics and Optical Communications, Beijing University of Posts and \\ Telecommunications, Beijing,100876, China \\ xzsang@bupt.edu.cn
}

\begin{abstract}
A multi-view content generation method for three-dimensional (3D) display without depth-reversed area is demonstrated. The viewing zone periodicity of an autostereoscopic display is utilized to eliminate the depthreversal by adjusting the light field alignment in the 3D light field. The conditions that a multi-view sequence should satisfies to form a depthreversal-free sequence are given and detailed processes for converting the multi-view sequence into a depth-reversal-free sequence are presented. Experimental results show that the depth-reversed area is well eliminated, and the smooth motion parallax can be achieved when the viewer moves in front of the display within a $60^{\circ}$ viewing angle. The number of presented perspectives can be 8 times more than the viewpoint number of the autostereoscopic display with the help of viewing zones integration.
\end{abstract}

Keywords: Autostereoscopic display; Three-dimensional image processing; Light field display

\section{Introduction}

With the development of the three-dimensional (3D) display, multi-view autostereoscopic displays are getting closer and closer to common customers. The autostereoscopic display with larger size, higher resolution and denser viewpoints were demonstrated [1-4]. The autostereoscopic 3D effect is typically achieved by adding a lenticular sheet or a parallax barrier in front of a common LCD display. The parallax barrier technology seriously reduces the brightness of images, so it is replaced by lenticular sheet technology in most products. In the lenticular based autostereoscopic display, the role of the lenticular lens is to magnify and transfer the information of specific pixels to a designated position [5]. The basic principle of lenticular based autostereoscopic display is shown in fig. 1, which is a five-viewpoint autostereoscopic display. For a given lenticular lens, it covers five columns of pixels, the covered columns of pixels emit light rays via the lenticular lens form a primary viewing zone, moreover, the columns of pixels covered by other lenticular lens also emit light rays via this given lenticular lens which makes repetitive viewing zones in front of the display. This periodicity extends the field of view of an autostereoscopic display and provides the immersive glasses-free 3D effect for multiple viewers at different positions. However, when two eyes fall into the depth-reversed area, the red blocks shown in fig. 1, the depth sense is reversed and the motion parallax becomes discontinuous. The viewing quality is significant reduced by the depth-reversed area. 


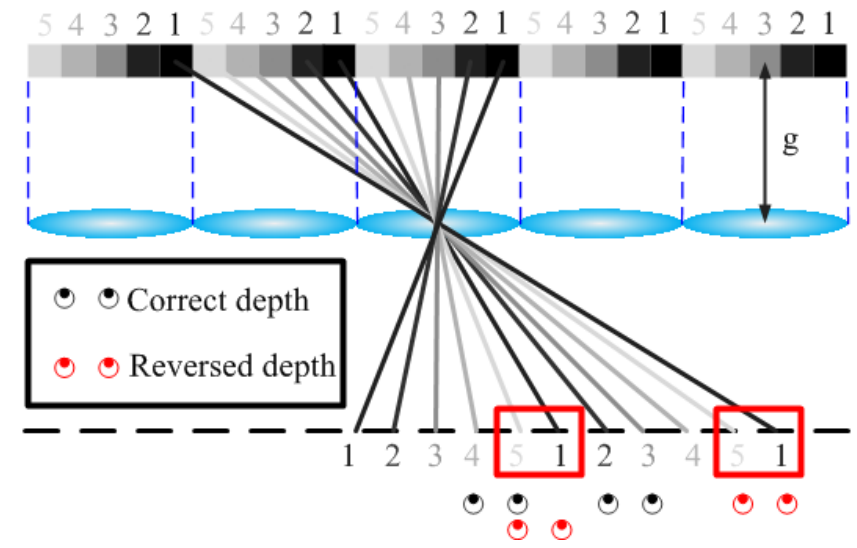

Fig. 1 The basic principle of autostereoscopic display

The depth-reversal is considered as an inherent disadvantage of the lenticular based or the parallax barrier based autostereoscopic display. To eliminate the depth-reversed area, the viewer's position is tracked and according to the viewer's position the displayed content [4] or the parallax barrier $[6,7]$ is changed in real time to make sure that the viewer never falls into the depth-reversed area. However, a dynamic parallax barrier increases the hardware complexity and the human tracking method is limited to only several viewers. Recently, instead of avoiding eyes of viewers fall into the depth-reversed area, a software-defined light field edit method was proposed to improve visual quality in the depth-reversed area [8], the basic principle of the method is to make the motion parallax of a light field at the depthreversed area as smooth as possible. So the light fields of adjacent viewing zones are seamless composited with light field stitching to make the maximum content smoothness at the depthreversed area. The psychological visual effect is improves, however, the method expands the depth-reversed area, redistributes the depth-reversal to more viewpoints and the correct scene structure is deformed due to image stitching.

As we known, previous researches generated the light field without connecting the multiview contents with the viewing zone periodicity. To make a difference, taking viewing zone periodicity into consideration and according to the same basic principle as [8], a simple but effective method based on light field alignment is proposed to completely eliminate the depthreversal and present interesting 3D effect different from previous works. An example is shown in our previous work [9], an elemental image coding method for integral imaging is introduced. According to a given depth map, the elemental image is shifted and packed repeatedly on the LCD panel. Thus the elemental images' periodicity is matched well with the viewing zone periodicity, thus the light fields at depth-reversed area are aligned and the depthreversal is completely eliminated. In this paper, the image coding method in [9] is generalized and improved with the help of light field theory. The basic principle of light field alignment is analyzed and a detail guide for light field alignment is deduced mathematically. According to the guide, not only a constant picture but also a series of multi-perspective images can be edited without depth-reversal and the number of viewpoints of a light field can be larger than an autostereoscopic display.

In the next section, 3D light field is briefly introduced and the viewing zone periodicity is explained with a visualized light field representation. The idea of depth-reversal-free light field alignment method is analyzed in section 3. 3D effects without depth-reversal in the autostereoscopic display are experimentally demonstrated in section 4. 


\section{3D Light field and viewing zone periodicity}

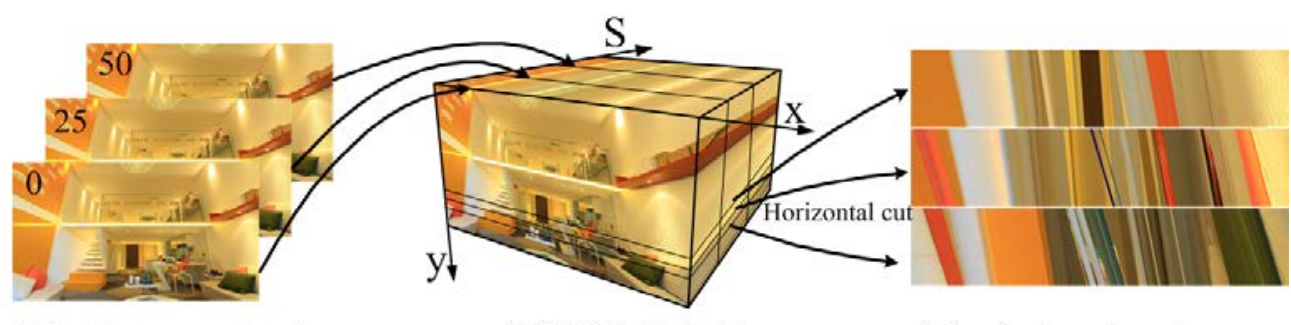

(a) Multi-perspective images

(b) 3D light field

(c) epipolar plane images

Fig. 2 3D light field

The light field was proposed by Gortler et al and Adelson et al [10,11], which can be expressed with a four-dimensional (4D) function called the Lumigraph or plenoptic function. The content that generated for the lenticular based autostereoscopic display should satisfy the multi-view stereo constraint: horizontal parallax only without the vertical displacement. So the $3 \mathrm{D}$ light field can be used in 3D image processing without loss of the generality. The concept of the 3D light field is presented in fig. 2. The 3D light field shown in fig. 2 (b) is assembled from a dense set of multi-perspective images. Different 2D sections of the 3D light field results in different 2D images that reflect various features of the 3D scene. An $x-y$ cut at particular parameter $S$ extracts the original $S^{\text {th }}$ multi-perspective image, and an $x-S$ cut leads to the epipolar plane image (EPI), which shows gradual changing of the multi-perspective images in the light field and presents smooth structures. The slope of the smooth structure is proportional to the depth of the corresponding 3D scene [12].

Based on the light field representation, the viewing zone periodicity can be vividly shown as the repeated EPI in fig. 3. One EPI represents one complete viewing zone. When the viewer moves left or right in front of the 3D display, viewing zones are periodically appeared just as the replicas of $3 \mathrm{D}$ light field are connected. As the same as fig. 1, when two eyes fall into different viewing zones, the depth-reversal occurs as the red eyes in fig. 3 . It is clear that the light field is not smooth at the junctions, and depth-reversal and image flipping occur.

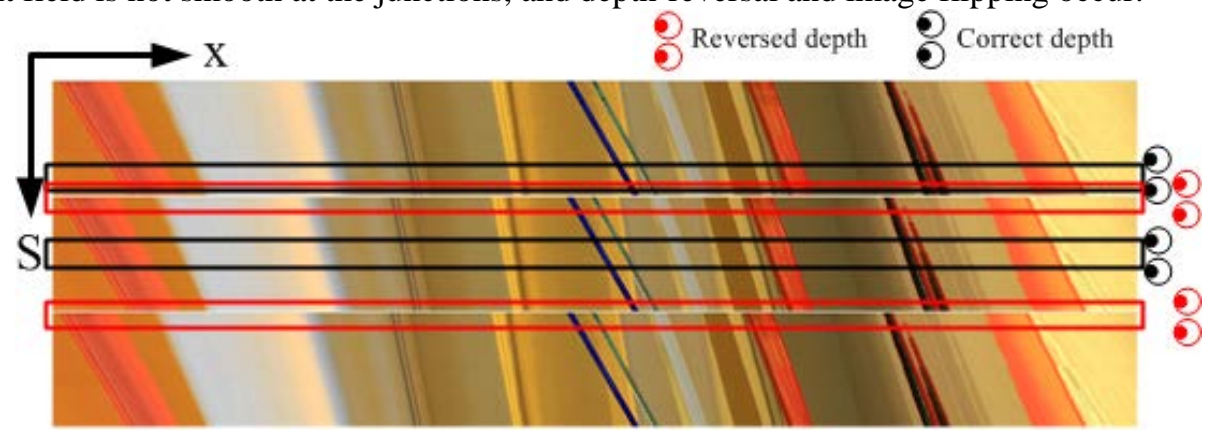

Fig. 3 Viewing zone periodicity with light field representation, viewing zones are periodically appeared just as the replicas of light field are connected.

\section{Adjustment of the light field alignment}

\subsection{Basic principle of the light flied alignment}

According to the analysis in section 2, the depth-reversal can be eliminated if junctions between the light field and its replicas are smooth, and a heuristic example is shown in fig. 4. Given an source light field $\ell(x, y, s)$, to eliminate the depth-reversal, it is firstly adjusted to the sub light field $\ell_{s}(x, y, s)$ with global shearing [8,13], and then the replicas of 
$\ell_{s}(x, y, s)$ are put to fill in the displayed light field with an interval $L$ as the red dotted lines,

$$
L=\frac{N}{|\tan \theta|}
$$

where $N$ is the viewpoint number of the autostereoscopic display, $\theta$ is the included angle with positive $\mathrm{x}$-axis. The source light field $\ell(x, y, s)$ should be a periodic function with a period $N$ for variable $S$,

$$
\ell(x, y, s)=\ell(x, y, s+N)
$$

according to eq. (2) the source light field should be a periodic function and the period is the viewpoint number of the autostereoscopic display.

The parallax value $d$ between two views is computed as,

$$
d=\frac{d x}{d S}=\frac{1}{d S / d x}=\frac{1}{\tan \theta}
$$

where the sign of $\tan \theta$ indicates positive or negative parallax. Taking eq. (3) into eq. (1) the relationship between the sub light field interval and displayed depth as shown in eq. (4) can be obtained,

$$
L=N|d|
$$

and according to eq. (4), the smooth light field alignment can be realized at the arbitrarily displayed depth with the corresponding parallax $d$.

Equation (2) gives the precondition of a source light field to realize a depth-reversal-free 3D effect, so the example light field in section 2 can not be used for depth-reversal eliminating. Equation (3) give a guide to shear the source light field according to a given displayed depth and then the sub light field is put into the displayed light field with an interval computed by eq. (1) or eq. (4). The detailed sub light field generation and light field alignment methods are shown in section 3.3. By meeting those conditions, the displayed light field becomes periodic and can be aligned smoothly at the light field junctions, and the depthreversal can be removed.

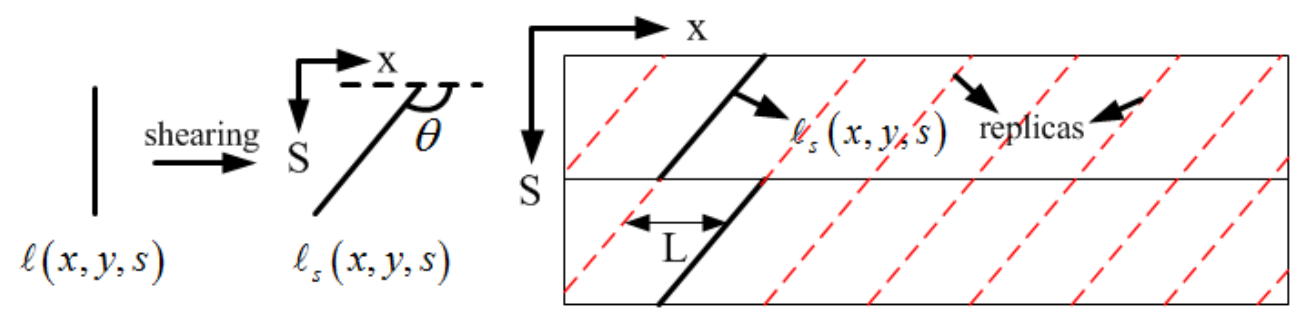

Fig. 4 Heuristic example of light field alignment, the source light field is sheared and packed repeatedly according to $L$ to make the light field aligned.

In our previous work [9], elemental images are repetitive, so the same content exists in each perspective in the corresponding source light field. For variable $S$, the source light field is a constant function,

$$
\ell(x, y, s)=C(x, y), s=1,2, \ldots
$$

Where $C(x, y)$ is the elemental image, compared with eq. (2), now the source light field is generalized to a periodic function and more dynamic effect can be realized with a periodic function than a constant function. 


\subsection{Multi-viewing-zone integration}

Another improvement of the proposed method is multi-viewing-zone integration, which utilizes a group of successive viewing zones to present a whole light field, and the perspective number of the displayed light field can be increased. As shown in fig. 5 (a), a period source light field with $R$ multi-perspective images is given, where $R$ is a positive integer. Assuming that there are $\mathrm{N}$ viewpoints for an autostereoscopic display, and it is impossible to display the content with more perspectives than $\mathrm{N}$ in traditional coding method. Here, with the help of multi-viewing-zone integration, a light field with more perspectives than the autostereoscopic display can be presented completely within a group of successive viewing zones, for an example $R=4 N$ in fig. 5 . As shown in fig. 5 (b), the sub light field is generated with global shearing from fig. 5 (a) and divided into segments, each segment has $\mathrm{N}$ perspectives. Then the segments are put into an EPI in turn, in other word the segments are put into the same viewing zone. According to the basic principle in section 3.1, the included angle $\theta$ and the interval $L$ are linked by eq. (1). Moreover, because the source light field is a period light field, it can be divided into infinite segments, thus the displayed light field can be filled up with segments of sub light field as shown the EPI in fig. 5 (c). Here, $R / N=4$ successive viewing zones are integrated as a whole to display all the perspectives of the source light field, and the red dotted box shows a whole of the integrated sub light field.

$$
\mid
$$

(a)

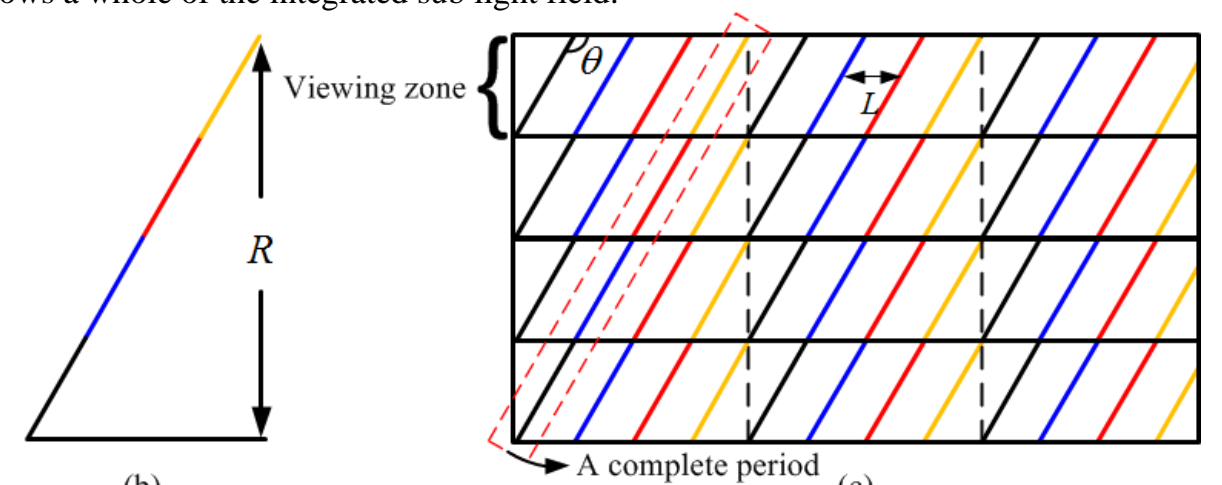

(c)

Fig. 5 The concept of multi-viewing-zone integration, (a) source period light field with $\mathrm{R}$ multi-perspective images ( $\mathrm{R}=4 \mathrm{~N}$ ), (b) sub light field, (c) light field alignment within a group of successive viewing zones

\subsection{Light field alignment with complex depth structure}

In previous sections, the slope of the sub light field is a constant, and the parallax is also a constant. The light fields are linear and the content is displayed at a given depth plane according to the light field included angle $\theta$. In this section, the light field generation method is expanded to the scene with the complex depth information. The global shearing process is replaced by light field shifting. As shown in fig. 6 (a) to fig. 6 (c), For a period source light field and a structured depth map for the 3D display, the source light field should be shifted according to the parallax between adjacent perspectives, and the displayed depth $z(x, y)$ can be converted to parallax $d(x, y)$ as,

$$
d(x, y)=\frac{z(x, y)}{V_{o}+z(x, y)} e
$$

where $V_{o}$ is the optimum viewing distance, $e$ is the pupil distance, and the positive distance $Z$ is defined as the depth inside the display screen.

The sub light field in fig.6 (c) can be obtained according to, 


$$
\begin{gathered}
x_{0}=x, x \in\left[\frac{W \text { _display }}{2}-\frac{W \text { _lightfield }}{2}, \frac{W_{\text {_display }}}{2}+\frac{W \text { _lightfield }}{2}\right] \\
x_{s+1}=x_{s}+d\left(x_{s}, y_{s}\right), \mathrm{s}=[0, \pm 1, \pm 2, \ldots]
\end{gathered}
$$

where $x$ is the pixel coordinate of each perspective images in the source light field, and the pixel at $x$ in the $s^{\text {th }}$ perspective image is shifted to $x_{s}$. Equation (8) gives the parallax relation between adjacent perspective images in the sub light field recursively. And eq. (7) sets the origin of the light field shifting to the central viewing position, $W_{-}$display is the width of an autostereoscopic display, $W$ _lightfield is the width of the source period light field in fig. 6 (a). The recursive process is carried out until $x_{s}$ is out of the pre-defined 3D display range in order to fill in the displayed light field as shown in fig. 6 (c). According to section 3.2, the sub light field is divided into segments, then the segments are put into one viewing zone with the same $\mathrm{x}$ coordinate as they are in the sub light field to obtain the displayed light field as shown in fig. 6 (d). Figure 6 (e) figures out a group of successive viewing zones to show the alignment effect of the light field.

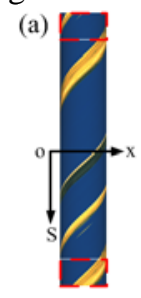

(b)

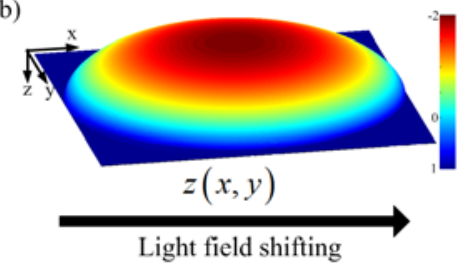

(d)

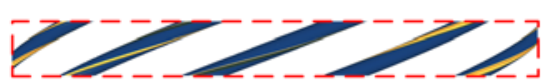

(c)

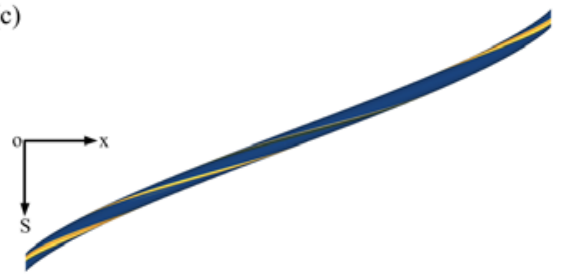

(e)

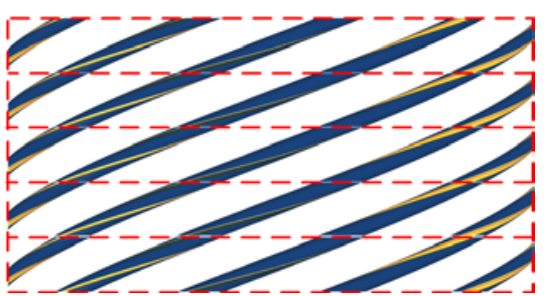

Fig. 6 Light field generation with the complex structured depth map, (a) source period light field, (b) structured depth map, (c) sub light field, (d) display light field, (e) light field alignment within a group of successive viewing zones

The eq. (7) and eq. (8) put forward the all-purpose formulas for light field alignment not only for complex depth structure in section 3.3 but also for constant depth in section 3.1 and section 3.2. For the depth is constant, the parallax $d\left(x_{s}, y_{s}\right)$ becomes a constant $d$ and the eq. (8) becomes,

$$
x_{s+1}=x_{s}+d
$$

thus the shifted light field is linear, and the intervals $L$ between segments are consistent,

$$
L=x_{s+N}-x_{s}=x_{s}+N d-x_{s}=N d
$$

which is the same as eq. (4).

\section{Experiments and discussion}

Experiments are carried out to demonstrate our light field alignment method. Two source light fields are used in the experiments, as shown in fig. 7. The earth and a letter " $\mathrm{C}$ " both rotate by 360 degrees, which forms period light fields naturally. The earth sequence has 400 perspectives while the letter " $\mathrm{C}$ " sequence has 224 perspectives. Some perspectives are shown in fig. 7 (a), and some examples of EPI with corresponding lines in fig. 7 (a) are shown in fig. 
7 (b). The parameter of the autostereoscopic display is shown in Table 1.The display is a 50 inch 4K LCD display, a sub pixel means an individual R, G or B pixel, and each lenticular covers 7 sub pixels. There are 28 viewpoints for the display configuration, and the pixel arrangement method can be found in our previous work [14].
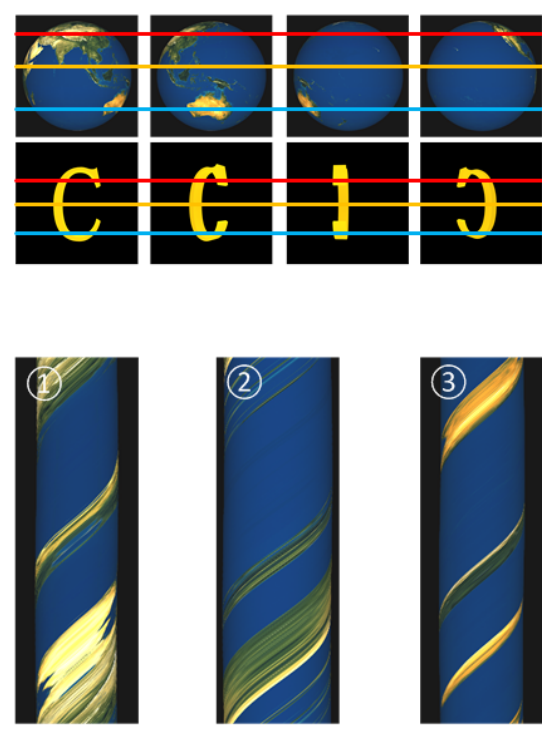

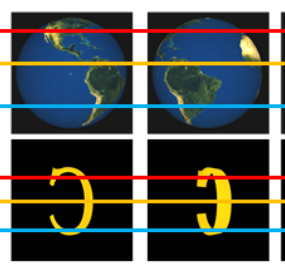

(a)
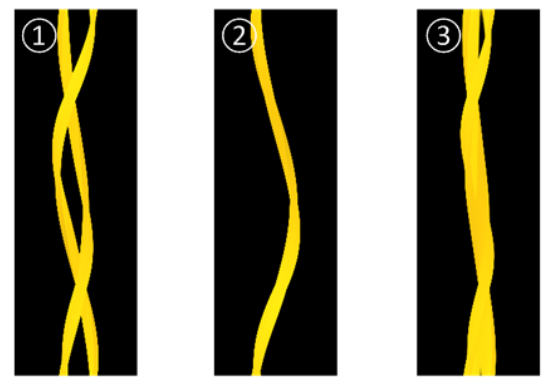

(b)
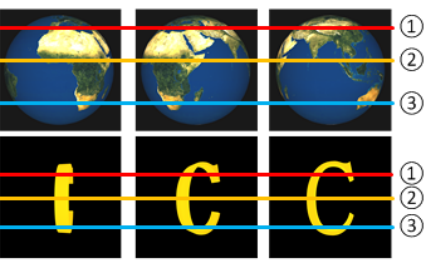

Fig. 7 Source light field, (a) images of different perspectives in the source light field, (b) EPIs of corresponding lines in (a).

Table 1 Parameters of the display configuration

\begin{tabular}{|c|c|c|c|}
\hline 2D resolution & Sub pixel pitch & Lenticular pitch & Viewpoints \\
\hline $3840 * 2160$ & $0.0961 \mathrm{~mm}$ & $7 * 0.0961 \mathrm{~mm}$ & $7 * 4$ \\
\hline
\end{tabular}

\subsection{D effect with and without light field alignment}

In the first experiment, the conditions that a light field should satisfies to realize light field alignment are discussed, respectively. According to section 3, to align the displayed light field, the source light field should be a periodic function and the sub light field interval is computed with eq. (1), eq. (4) or the all-purpose formulas eq. (7) and eq. (8).

In fig. 8 (a), only half perspectives of the earth sequence are used as the source light field, in other words, in this source light field the earth only rotates 180 degrees which breaks the periodicity of the source light field. The EPIs in fig. 8 (a) shows the unsmooth motion parallax without periodic source light field, the light field cracks appear as shown in the red boxes in fig. 8 (a) when the first image meets the last image in a light field. Video 1 gives the experiment result, the earth's texture jumps at the cracks and make the 3D effect bad.

Figure. 8 (b) gives the result of the 3D effect without considering the intervals between sub light field according to the proposed equations, as shown in fig. 8 (b), the light fields are misaligned at the junction of two adjacent viewing zones, video 2 gives the experiment result, depth-reversed area appears and make the 3D effect bad.

The 3D effect with the proposed method is shown in fig. 8 (c), the light field is well aligned, thus the motion parallax is smooth and there is no depth-reversed area when viewers walking around in front of the display. The visualize experiment results can be seen in video 1 , video 2 and video 3, respectively. And the experiment results show that only the conditions proposed in above sections are satisfied, depth-reversal can be completely eliminated. 


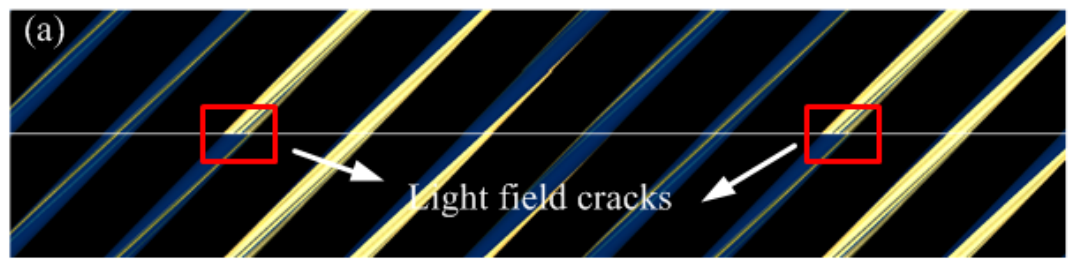

Viewing

zone 1

Viewing

zone 2

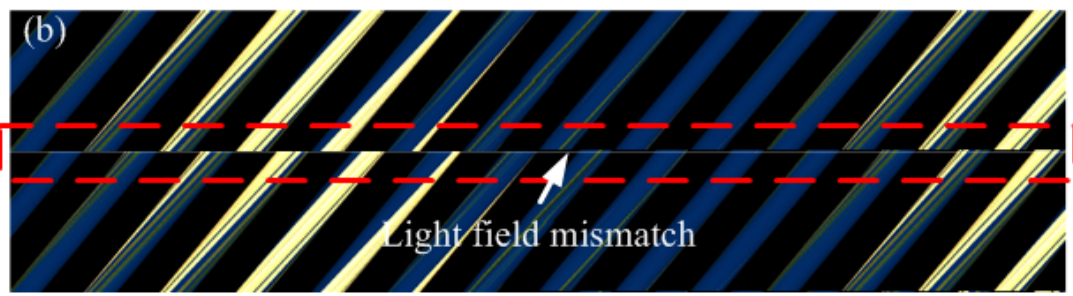

Viewing zone 1

Viewing zone 2

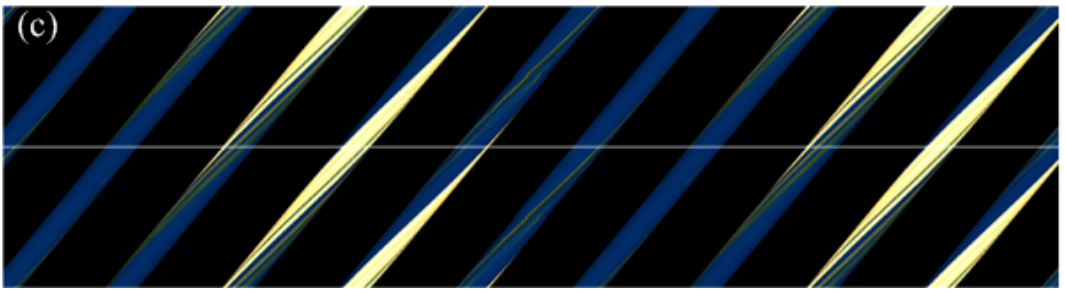

Viewing zone 1

Viewing zone 2

Fig. 8 (a) Autostereoscopic display without periodic source light field (see Video 1), (b) Autostereoscopic display without considering the intervals between sub light field (see Video 2), (c) Autostereoscopic display with the proposed method (see Video 3).

\subsection{Depth-reversal-free autostereoscopic display with the complex depth structure}

With the method proposed in section 3, the displayed light field is generated as shown in fig. 9, according to the complex depth structure in fig. 6 (b) and the sub light fields in fig. 7 (a). In the experiment, 3D ellipsoid structure out of the screen is formed by the rotating "C" and the flat background inside the screen is formed by the rotating earth. In Video 4, there is no depthreversal and image flipping when the viewer walking around in front of the display. The earth and the " $\mathrm{C}$ " change smoothly and continuously. The viewing angle is 60 degrees.

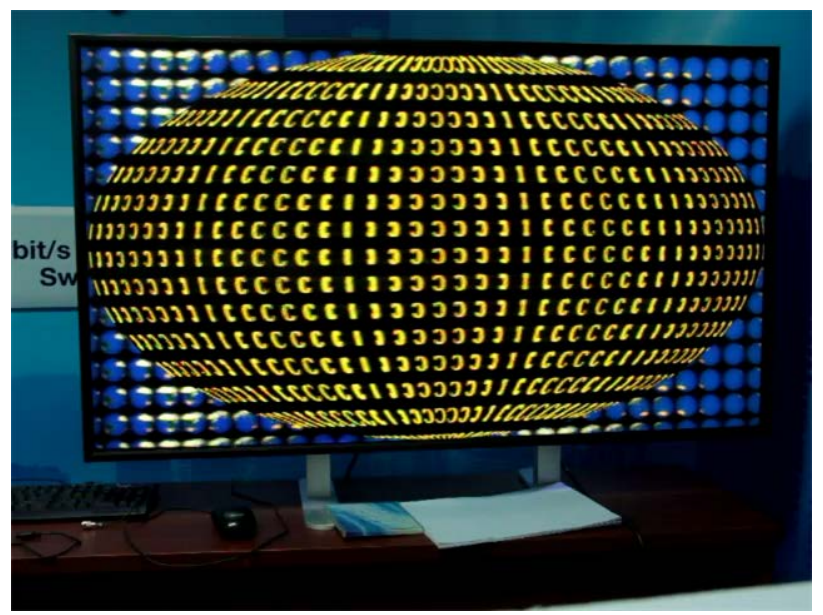

Fig. 9 Depth-reversal-free autostereoscopic display with the complex depth structure (see Video 4) 


\subsection{Multi-viewing-zone integration}

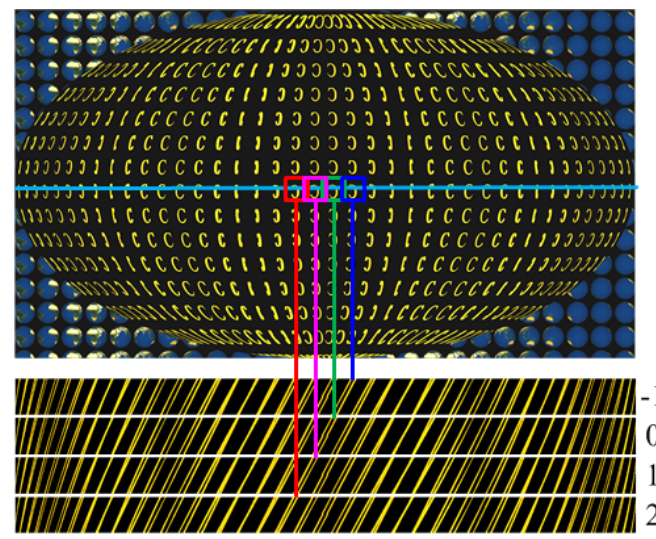

(a)

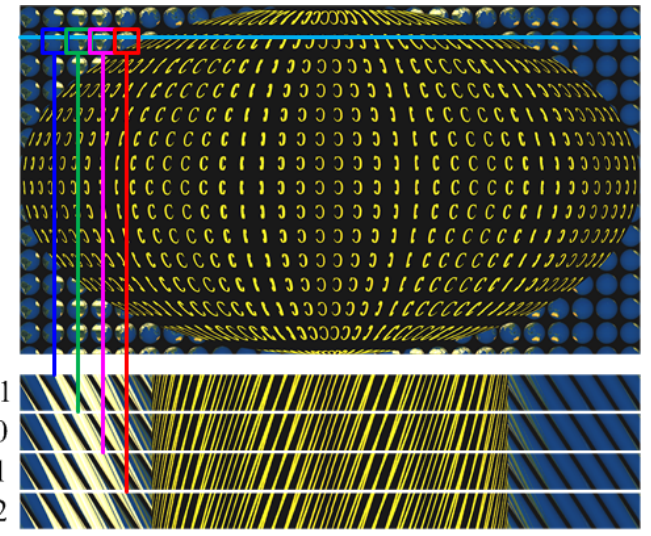

(b)

Fig. 10 Multi-viewing-zone integration, (a) viewing zones integration of the letter "C" and (b) viewing zones integration of the earth

One perspective of the displayed light field and the corresponding EPIs of four viewing zones are shown in fig. 10. In fig. 10 (a), starting at viewing zone 0 and staring at the " $C$ " with green box, when moving left in front of the 3D display, the viewer enters the viewing zone -1 and the "C" translates and rotates into the blue box. When moving right, the viewer enters viewing zone 1 and the "C" translates and rotates into the pink box. The viewer will enter viewing zone 2 and the "C" will translate and rotate into the red box if the viewer continue moves right. In fig. 10 (b) when the viewer moves right, the earth translates and rotates from blue box into red box. It is clear that the "C" and the earth present different perspectives in successive viewing zones and the successive traditional viewing zones are integrated into an entire view zone, which can present more perspectives of a 3D scene many times than a traditional viewing zone in the same autostereoscopic display. In the experiment, a 28-viewpoint autostereoscopic display is used to present the earth with 400 perspectives and the "C" with 224 perspectives.

\section{Conclusion}

With 3D light field analysis, the principle of depth-reversal-free 3D display is demonstrated. By implementing the proposed adjustment of the light field alignment, the periodicity of the displayed light field is fitted well with the viewing zone periodicity of the autostereoscopic display, which makes the traditional depth-reversed areas also have a correct 3D depth sense, and a smooth motion parallax is achieved at the same time. Although the source light field is limited to periodic function and the size of images in the source light field should be smaller than the sub light field interval $L$, it finds another way to overcome the depth- reversal problem in lenticular based autostereoscopic display and shows exciting 3D effect which is different from previous image generation method for autostereoscopic display. Moreover, the proposed method is possible to make an extension of the number of viewpoints for an autosteroscopic display with multi-viewing-zone integration. Experiment results demonstrate the effectiveness of the proposed method, and an amazing 3D display is recorded.

\section{Acknowledgment}

This work is partly supported by the "863” Program (2015AA015902), the National Science Foundation of China (61575025), the fund of the State Key Laboratory of Information Photonics and Optical Communications, and the Program of Beijing Science and Technology Plan (D121100004812001).

\section{References}

1. N. S. Holliman, N. A. Dodgson, G. E. Favalora, and L. Pockett, "Three-dimensional displays: A review and applications analysis,” IEEE Trans. Broadcast 57(2), 362-371 (2011). 
2. X. Sang, F. C. Fan, C. C. Jiang, S. Choi, W. Dou, C. Yu, and D. Xu, "Demonstration of a large-size real-time full-color three-dimensional display, ” Opt. Lett. 34(24), 3803-3805 (2009).

3. X. Gao, X. Sang, X. Yu, P. Wang, X. Cao, L. Sun, B. Yan, J. Yuan, K. Wang, C. Yu, and W. Dou, “Aberration analyses for improving the frontal projection three-dimensional display,” Opt. Express 22(19), 23496-23511 (2014).

4. X. Yu, X. Sang, S. Xing, T. Zhao, D. Chen, Y. Cai, B. Yan, K. Wang, J. Yuan, C. Yu and W. Dou, "Natural three-dimensional display with smooth motion parallax using active partially pixelated masks," Opt. Commun 313, 146-151 (2014).

5. J. Hong, Y. Kim , H. J. Choi, J. Hahn, J. H. Park, H. Kim and B. Lee. “Three-dimensional display technologies of recent interest: principles, status, and issues [Invited] ”. Applied optics 50(34), H87-H115 (2011).

6. T. Peterka, R. L. Kooima, D. J. Sandin, A. Johnson, J. Leigh and T. A. DeFanti, "Advances in the dynallax solid-state dynamic parallax barrier autostereoscopic visualization display system,”, IEEE Transactions on Visualization and Computer Graphics 14(3), 487-499 (2008).

7. S. Y. Yi, H. B. Chae and S. H. Lee, "Moving parallax barrier design for eye-tracking autostereoscopic displays," IEEE 3DTV Conference on the True Vision-Capture, Transmission and Display of 3D Video, 165168, (2008).

8. S. P. Du, P. Didyk, F. Durand, S. M. Hu and W. Matusik, "Improving visual quality of view transitions in automultiscopic displays,” ACM Transactions on Graphics, 33(6), 192 (2014).

9. X. Yu, X. Sang, X. Gao, Z. Chen, D. Chen, W. Duan and D. Xu, "Large viewing angle three-dimensional display with smooth motion parallax and accurate depth cues," Optics express, 23(20), 25950-25958 (2015).

10. Steven J. Gortler, R. Grzeszczuk, R. Szeliski and M. F. Cohen, “The lumigraph,” Proceedings of the 23rd annual conference on Computer graphics and interactive techniques, ACM (1996).

11. Edward H. Adelson and James R. Bergen, "The plenoptic function and the elements of early vision," Computational models of visual processing (1991).

12. C. Kim, H. Zimmer, Y. Pritch, A. Sorkine-Hornung and M. H. Gross, "Scene reconstruction from high spatioangular resolution light fields,” ACM Trans. Graph., 32(4), 73-1 (2013).

13. S. Xie, P. Wang, X. Sang, C. Li, W. Dou and L. Xiao, "Depth-tunable three-dimensional display with interactive light field control," Optics Communications, 371, 166-172 (2016).

14. X. Yu, X. Sang, D. Chen, P. Wang, X. Gao, T. Zhao and W. Dou, "Autostereoscopic three-dimensional display with high dense views and the narrow structure pitch,” Chinese Optics Letters, 12(6), pp. 060008 (2014). 